\title{
HOF: Adjusting the Rule-Based \\ Safety/Managed Safety Balance and Keeping Pace with a Changing Reality
}

\author{
Caroline Kamaté
}

\begin{abstract}
It is commonly acknowledged among at-risk industrial sectors that improvement in safety performance requires better consideration of HOF. Tensions and even contradictions exist between work and organisation as theoretically understood, and the reality of the shop floor, with its constraints, its power games and more. HOF specialists are, in some ways, the voice of reality in complex sociotechnical systems such as at-risk organisations. The HOF approach provides, at all levels, 'adjustment loops' to promote safe and efficient human activity and contribute to the business whole performance. However, the way HOF are structured varies widely depending on organisations and the expectations in terms of both impact and sustainability are not always met. This final chapter briefly summarizes and discusses some of the axes for improvement previously presented in the book.
\end{abstract}

Keywords Reality • 'Organising' · HOF dynamic loop

\section{Introduction}

It is nowadays generally accepted that if the safety strategy of a company is to be improved, a further step must be taken in the consideration of human and organisational factors (HOF). Thus, requests on the topic of HOF abound. However, the way HOF structures are organised denotes a heterogenous and fragmented HOF landscape, according to the company and even within companies. Furthermore, what is implemented does not always meet the expectations in terms of impact and continuity, hence the safety outcomes, and HOF actors sometimes deplore a lack of leeway and integration of their contribution at the organisation's highest levels.

Thus, although (almost) everybody is convinced about the importance of considering human and organisational factors for safety, in most industries there is a feeling of dissatisfaction or even frustration. What are the conceptual, structural and func-

\author{
C. Kamaté (凶) \\ FonCSI, Toulouse, France \\ e-mail: caroline.kamate@foncsi.org \\ (C) The Author(s) 2020 \\ B. Journé et al. (eds.), Human and Organisational Factors, \\ SpringerBriefs in Safety Management, \\ https://doi.org/10.1007/978-3-030-25639-5_16
}


tional levers for an implementation of HOF approaches that efficiently contribute to safety performance? Based on the work presented in the previous chapters of the book, this synthesis is an attempt to summarize and build on the main findings.

\section{HOF Approaches for Capturing Reality}

Adopting a HOF approach means examining human work within the organisation, notably beyond the framework, rules and procedures that govern it. Indeed, beyond the prescriptions, depending on the context and its unforeseen circumstances, employees adapt their activity, which is not limited to the prescribed work, to 'do their job'. In the same way, the organisation is above all a structure, with an organisation chart and rules which frame its functioning as well as accounts that must be rendered to the external stakeholders. But an organisation is also a process, it is continuously under construction: it is the living and dynamic product of a set of interactions and social regulations. It is the outcome of an actual 'organising' work carried out daily by all actors, including managers through the arbitrations they are led to do. This organising is both vertical (between hierarchical levels) and horizontal (management of internal and external interfaces).

This hiatus between a theoretical and normative view of how work should be done or how organisations should function, and what really goes on in situation, is at the core of HOF. HOF specialists have the duty to always consider reality and its constraints, and to 'ring the reality bell' at all levels of the organisation. They may somehow be considered as 'providers of reality'.

\section{Support 'Organising'}

There are some essential conditions for allowing HOF people to fulfill their functional mission. They must be connected to the shop floor and be able to feed back reality of work to the highest levels of the organisation. This means their words must not be censured, and managers should also be open to listen to bad news. The freedom of speech of HOF people is a number one priority and must be protected. HOF resources must be deployed wherever needed in the organisation to identify contradictory issues and support managers in their arbitrations and trade-offs to get the job done, and to promote interactions at all levels. Thus, they favorize constant 'organising'. 


\section{Work on the Gap between Expectations and Responses}

The way HOF specialists and senior managers respectively think about HOF, leads to differences in their perception of the role of HOF specialists. There is often a gap between some normative expectations of industry consisting in operational recommendations, quick and limited in scope interventions, and responses from $\mathrm{HOF}$ actors, both academics and practitioners, that are not that 'simple' ... As an example, if some human factors at the workplace level can quite easily be monitored by indicators, this is generally not the case anymore when the perimeter is extended to the level of the organisation, even more so with a changing dimension.

HOF specialists claim that an organisation is a socio-technical system with some human and organisational dimensions that mainly escape monitoring by indicators. Moreover, industrial companies, like other complex systems, are basically unstable. But despite the discourses about the impact of human and organisational factors and their hardly quantifiable features, for most company leaders, the organisation is seen above all as a techno-economic system, and it is mainly as such that they 'work it out'. They perceive the role of HOF specialists as being precisely to provide sociotechnical engineering, and not to remind them how difficult it is to do so. HOF are expected to restore a regulated safety ensuring 'normal stable' operations. Most regulators support this vision too, because it is easier to control and display, and reassuring for public opinion.

On this basis, there is de facto unrest among HOF specialists, and frustration from both sides. Since they cannot really fulfil the promise of socio-technical engineering, they eventually could be seen in a position of a 'permanently failing function'. However, they offer an 'imperfect remedy' for an 'imperfect reality', and they must constantly get back to work because this is a never-ending mission.

\section{Rebalance the $\mathrm{O}$ within the $\mathrm{F}$}

Although internal HOF structures regularly identify organisational causes when analysing unwanted events, they usually have much more influence on human factors than on organisational ones. There are several reasons that can explain this situation. One is the profile of HOF specialists - mostly ergonomists and psychologists - and their scope of intervention which is sometimes limited to health, safety and working conditions. Within that field, their expertise is fully recognized and the methods and tools they provide are operational and have proved efficient. However, work on organisational factors seems to remain within the purview of a different category of actors, mainly management specialists, who, unlike most HOF specialists who work in close interaction with front-line managers, operate at the decision-making level. To some extent, HOF specialists face a 'glass ceiling' that prevent them accessing the strategic levels of the company. This might undermine the efficiency of their approach due to their scope of action being limited to one part of the problem, and 
could significantly weaken their influence on strategic decisions. Unlocking this situation requires working at both conceptual and practical levels, starting with building a more integrated and consistent approach towards HOF around the cross-disciplinary concept of 'organising'. Promoting training of both executive committee members on the basis of HOF and HOF people in risk management, plus the presence of HOF experts within the executive board to provide support for decision-makers by forming binomen for example, are promising levers for safety and performance because they promote the reconciliation of these two worlds.

\section{Safety Alone Is not the Key}

Companies do not have only safety to manage and the priority given to safety does not always translate into reality. And, although this is a slight exaggeration, HOF can be described as knowledge-oriented while managers are solution-oriented. Consequently, at first glance $\mathrm{HOF}$ is not an issue for managers and must therefore be turned into a managerial issue (design, productivity...). Thus, to better mobilize around HOF, it is of strategic importance to demonstrate the connection of HOF to other key dimensions of business performance and to overall risk management rather than to safety only. The purpose of implementing HOF approaches is to promote safe and efficient human activity. But beyond safety and human efficiency, better consideration of HOF fosters the whole industrial performance through the integrative function of human activity. It also leads to the limitation of costs like human cost for performance (incidents or accidents, exhaustion, demobilization), costs due to late identification and catch up of design errors... Rather than the safety one, hanging this banner might give HOF people better chance to get attention from top managers. Once 'inoculated', once convinced sometimes by leading examples, the will of a few top-managers might facilitate openness to HOF from the whole organisation and support from the direction. The HOF policy must then translate into strategic piloting tools and the deployment of HOF resources all along the organisation's key processes.

\section{Reinforce the Dialogue around HOF with External Stakeholders}

Depending on the industrial sector, consideration of HOF by the regulators varies widely, with the authorities supervising civil aviation probably the more advanced in that field. There is no doubt that the greater incorporation of HOF into the regulatory framework is a powerful lever for improving their consideration by companies, and therefore promoting efficient and sustainable HOF approaches. Nevertheless, the risk exists of becoming too prescriptive and of shifting too much towards the rule-based 
safety side, thus denaturing the very purpose of HOF which is to look beyond the rules. The emphasis should be placed on the need for industrial organisations and regulators to share HOF fundamentals and engage a discussion.

Better knowledge and acknowledgment of HOF by judges is also a paramount issue in view of the increasing judicialization of industrial disasters. This is reflected by the good reception given by the European Parliament to the concept of just culture in civil aviation.

More globally, better consideration of HOF in inter-organisational relationships requires an institutional work which represents a promising area for renewed safety approaches.

\section{Assume the Dual Objective of HOF Structuring}

The way HOF are structured in a company must serve objectives of two different natures. HOF must be well structured to achieve their primary functional purpose as reality sensors which largely consists in managing the gaps between the work as it is conceived and the work as it is done, and seeking to narrow the gap. For that purpose, HOF must be networked and finely inserted in processes where safety is 'manufactured'. Nevertheless, HOF structure also has a symbolic scope. Internally, it positions HOF within the organisation's culture, establishing the permanence of the HOF approach, its relevance and legitimacy. Regarding the external environment of the company, it stresses the importance given to safety, designates the stakeholders' interlocutors and publicizes compliance with explicit or implicit standards. This symbolic role requires a much more visible and homogenous structuration. The risk exists that company leaders, in their arbitrations, emphasise the symbolic role because it is more visible and appears the most 'profitable' in the short term. But they need to adopt both strategies and assume this duality. The idea is to use the symbolic structure as an entry and exit point, providing a framework to the functional role of $\mathrm{HOF}$, the stake being that HOF people can operate, benefiting from the resources and leeway they need.

\section{The HOF Virtuous Loop}

The importance of overcoming old models essentially based on rule-based safety is nowadays more widely acknowledged. Since hazardous industries, like any other complex systems, are dynamic and unstable socio-technical systems, the largest potential for progression lies on the side of managed safety, which could also be named initiative-based safety. By starting from the real work with emphasis on 'organising', HOF approaches promote, at all levels, 'loops of adjustment' between the top managerial models and the reality of work. By identifying paradoxes and supporting the consideration of the different points of view, the HOF loop seeks 
to turn tensions into opportunities for improvement, rather than becoming sources of blockage. The instability of industrial organisations must be accepted in order to anticipate and adapt to future changes. Uncertainty, risk and a certain incompleteness contribute to adaptation, while trying to find fixed solutions carries the risk for organisations of always fall behind the times in a constantly changing world. Thus, rather than aiming to achieve perfection at a given moment, the HOF loop participates in considering imperfection as an asset rather than a problem, therefore contributing to organisational agility.

To keep the HOF loop dynamic and successful, HOF specialists face great challenges. They must provide advice and support at the highest level of the organisation, while keeping in touch with the reality of the shop floor. Being close both to decisionmakers and to the workplace, maintaining the same interest for the work of all those who contribute to safety and the same quality of dialogue requires them to master different languages. They have to make decisions about the right battles to be fought, which managers should be supported in their arbitrations and which resources must be negotiated. Their voice is essential and must weigh in the conduct of technical and organisational changes. And, of course, they must continuously stay up-to-date with advances in cognitive, social and organisational sciences. All of this requires the top management to be open to HOF and to unconditionally support implementation of a consistent HOF approach.

Open Access This chapter is licensed under the terms of the Creative Commons Attribution 4.0 International License (http://creativecommons.org/licenses/by/4.0/), which permits use, sharing, adaptation, distribution and reproduction in any medium or format, as long as you give appropriate credit to the original author(s) and the source, provide a link to the Creative Commons license and indicate if changes were made.

The images or other third party material in this chapter are included in the chapter's Creative Commons license, unless indicated otherwise in a credit line to the material. If material is not included in the chapter's Creative Commons license and your intended use is not permitted by statutory regulation or exceeds the permitted use, you will need to obtain permission directly from the copyright holder.

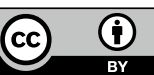

